\title{
A hybrid high-gain double-pass erbium-doped fiber amplifier with dispersion compensation feedback loop
}

\begin{abstract}
A new double-pass erbium-doped fiber amplifier (DP-EDFA) using chirped fiber Bragg grating (CFBG) is reported here showing a gain of $53.4 \mathrm{~dB}$ and noise figure (NF) of $5.36 \mathrm{~dB}$. The proposed amplifier at the same time provides dispersion compensation through the use of CFBG.
\end{abstract}

Keyword: Double-pass EDFA, Chirped fiber Bragg grating, Dispersion compensation 